\title{
Histiocytic Sarcoma: A CaSe of a 52-Year-old female With TWO SYNCHRONOUS PRIMARY MALIGNANCIES AT PRESENTATION
}

\author{
Ryan D. Gentzler, MD, Daron A. Kahn, MD
}

\section{Case Report}

A 52-year-old female with a past medical history of chronic obstructive pulmonary disease, coronary artery disease, and an 80-pack-year smoking history presented to the emergency room with complaints of right upper extremity weakness and imbalance while walking for the previous two days. She stated that the onset was abrupt and progressively worsening to the point where she could no longer lift her right upper extremity against gravity. She maintained the ability to move her hand and grasp, but admitted to decreased strength and decreased dexterity of her right hand. She denied changes in vision. She also denied having bowel or bladder incontinence. She has no known allergies and her only medications included occasional benzodiazepines for anxiety and acetaminophen/oxycodone for low back pain. Her family history was notable only for lung cancer, which was the cause of death of her mother.

On admission, the patient's vital signs were stable. She was afebrile, alert, and oriented to person, place, and time. Her physical exam was notable for profoundly reduced strength rated at $1 / 5$ in her proximal right upper extremity. Her distal forearm

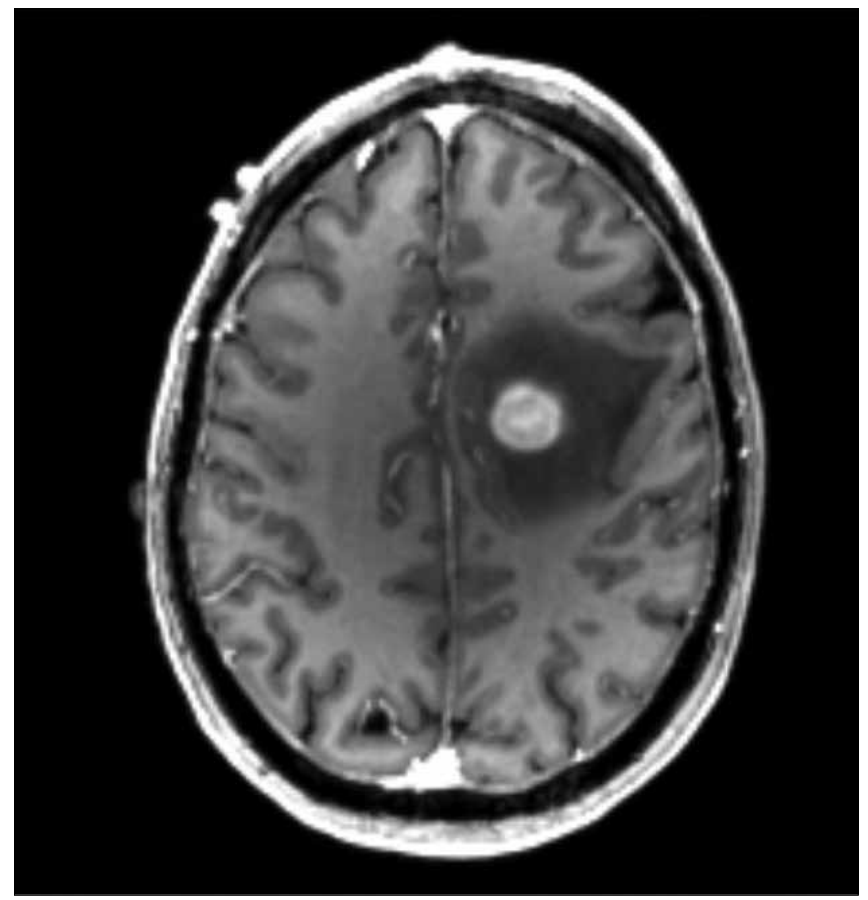

Figure 1. T1 weighted Brain MRI at the time of admission demonstrates a $1.6 \times 1.5 \times 1.7 \mathrm{~cm}$ solid enhancing mass centered on the left corona radiata with extensive surrounding vasogenic edema. T1 weighted Brain MRI at the time of admission demonstrates a $1.6 \times 1.5 \times 1.7 \mathrm{~cm}$ solid enhancing mass centered on the left corona radiata with extensive surrounding vasogenic edema.

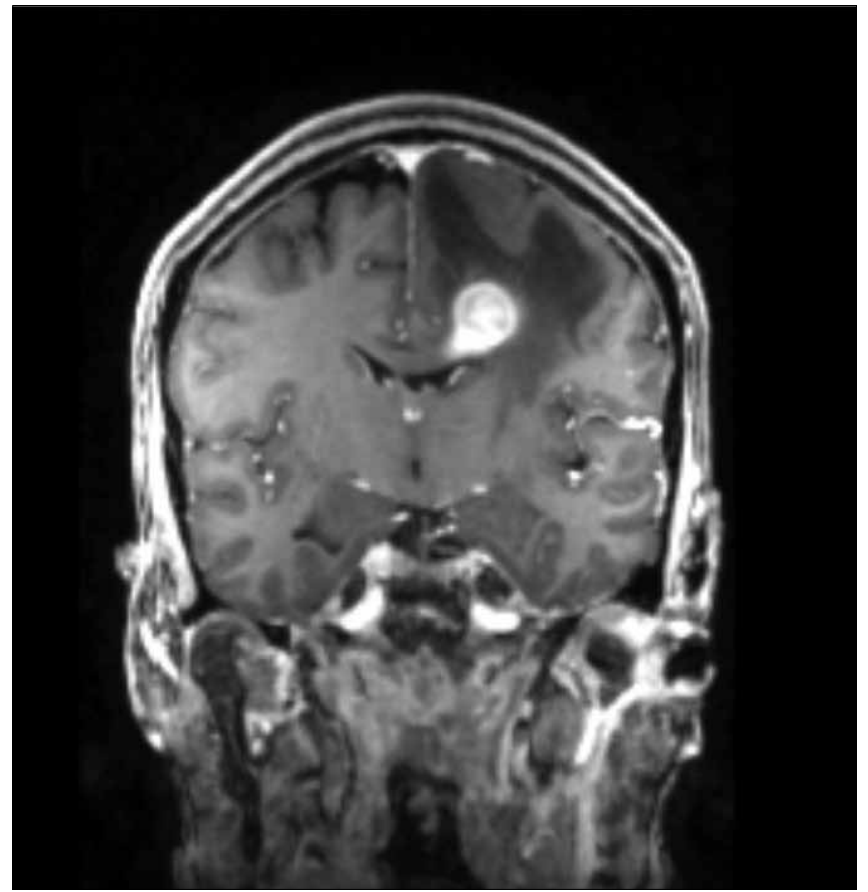

Figure 2. Axial view of T1 weighted MRI brain demonstrating left cerebral enhancing mass with surrounding edema

and hand were also reduced at 3/5 intensity. Her sensation in her right upper extremity was intact and symmetrical. All reflexes in her upper and lower extremities were $1+$.

A CT scan of her head revealed a large enhancing mass in the left parietal region. The patient was given levetiracetam $500 \mathrm{mg}$ twice daily for seizure prophylaxis and dexamethasone $4 \mathrm{mg}$ every 6 hours to reduce inflammation and edema surrounding the brain mass. A CT scan of the chest, abdomen, and pelvis was obtained for further workup and staging prior to surgery. This study revealed cavitating lung masses in the anterior left upper lobe and posterior right lobe, likely representing synchronous primary lung malignancies.

An MRI of the brain (Figures 1-2) on the same date revealed a $1.6 \times 1.5 \times 1.7 \mathrm{~cm}$ mass in the left centrum semiovale. After discussion with radiologists reading the image, the findings on MRI were concerning for primary CNS lymphoma and thought to be atypical findings for a metastatic brain lesion. A bronchoscopy performed the following day found an endobronchial lesion nearly occluding the left upper lobe, and biopsy yielded a diagnosis of squamous cell carcinoma.

Given the fact that the read on the MRI was inconsistent with metastatic disease from a possible lung origin and was more consistent with a primary CNS lymphoma, we felt it was 


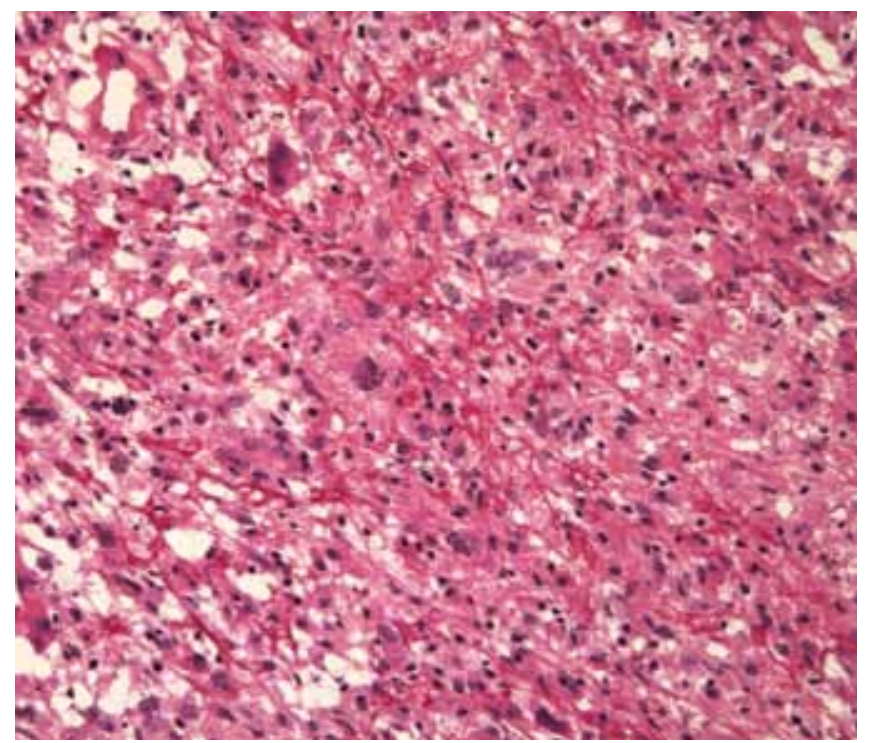

Figure 3. Light microscopy of brain lesion biopsy shows dense collection of histiocytes, many of which are atypical in appearance. (10x magnification, $H$ and $E$ stain)

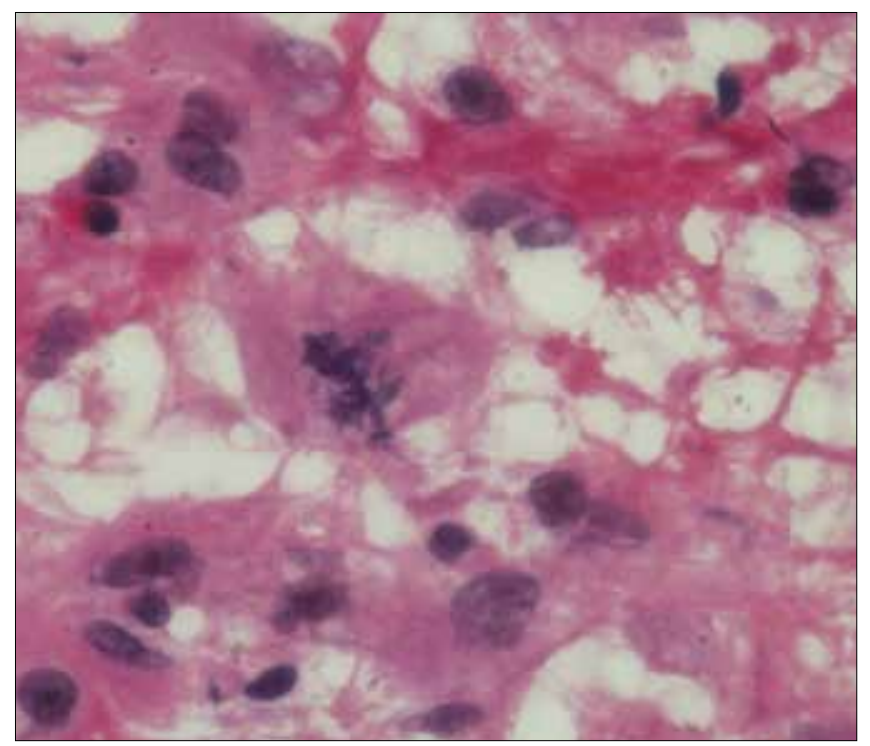

Figure 4. Atypical histiocyte seen at high power. (100x magnification, $H$ and $E$ stain)

necessary to proceed with a biopsy of the brain lesion before initiating treatment for lung cancer. During the course of the hospital stay, the patient was fortunate to have improved function of her right arm and her gait returned to normal. She was discharged with a steroid taper.

The final brain biopsy (Figures 3-4) results were obtained after discharge and confirmed the diagnosis of a second separate malignant process involving the brain. Evaluation of the biopsy in consultation with the pathology department at the NIH identified dense collections of histiocytes present within reactive brain tissue. Many of the histiocytes were atypical in appearance and stained CD68 and CD163 positive, which is consistent with a diagnosis of histiocytic sarcoma.

The patient initially responded well to steroids and regained most of the function of her upper and lower extremity and was discharged from the hospital after the initial workup. However, she was readmitted in less than one week with progressively worsening weakness and gait abnormalities and was found to have a slightly larger mass on imaging studies (Figure 5). Because of her severe neurological impairment, she was discharged to inpatient rehabilitation. Due to her functional status, she was not a candidate for chemotherapy, and she expired from severe hemoptysis that was likely secondary to erosion of her lung cancer through a pulmonary artery.

\section{Discussion}

The case presented above is noteworthy not only for the unusual presentation of synchronous primary neoplasms, but also for the diagnosis of a rare form of non-Hodgkin's lymphoma, histiocytic sarcoma (HS), which we believe to be a primary CNS histiocytic sarcoma. Histiocytic sarcoma is a rare malignancy that makes up less than $1 \%$ of all diagnosed non-Hodgkin's lymphoma and has mostly been reported only in small series of case reports. ${ }^{1}$ It is a malignancy of hematopoeitic cells resembling histiocytes but can be confused with similar looking T- and B-cell neoplasms. An extensive array of antibodies must be evaluated to differentiate HS from Langerhans Cell Sarcoma, diffuse large B-cell lymphoma, peripheral T-cell

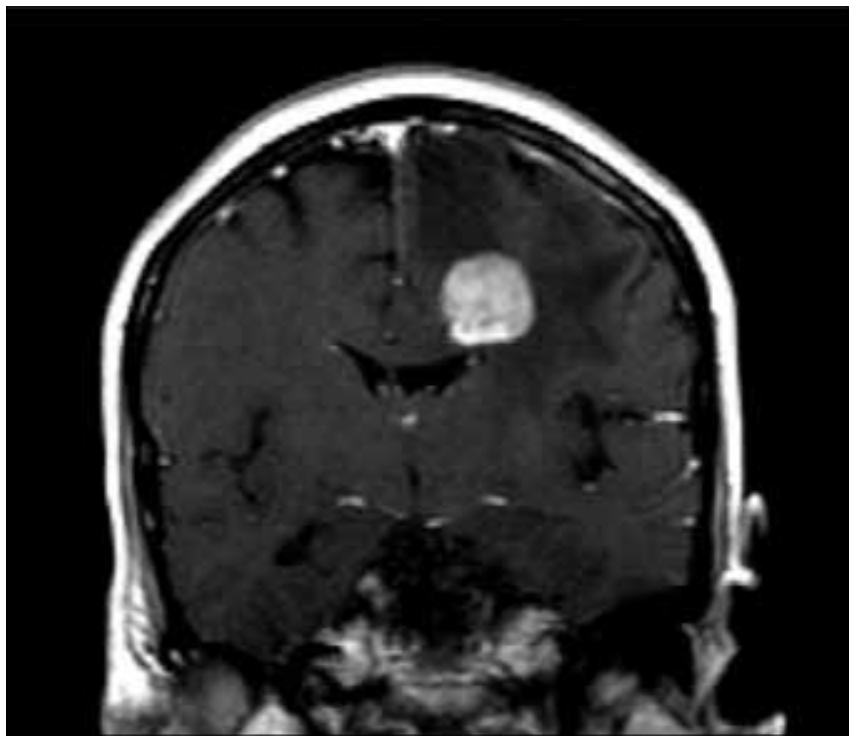

Figure 5. Axial view of $T 1$ weighted MRI of the brain 24 days after admission demonstrates a progressively enlarging mass (measuring $2.0 \mathrm{~cm} \times 1.6 \mathrm{~cm} \times 1.9 \mathrm{~cm}$ ) and worsening surrounding edema. This tumor progression is consistent with an aggressive nature of histiocytic sarcoma. 
lymphoma, acute lymphocytic lymphoma (ALL), metastatic undifferentiated carcinoma, and melanoma. ${ }^{1,2,3}$ Unlike other lymphomas, HS usually presents with extranodal involvement. About one-third of cases present within lymph nodes, and two-thirds are extranodal at presentation. ${ }^{2}$ Of the extranodal cases, about half present with skin lesions, and the other half with various sites of involvement including the intestinal tract, and rarely the central nervous system (CNS). ${ }^{2,45} \mathrm{HS}$ is usually an aggressive malignancy that presents with constitutional symptoms such as fever and weight loss and symptoms related to organ involvement such as pancytopenia, bowel obstruction, rash, and subcutaneous nodules. ${ }^{2}$ When diagnosed, HS is most often at an advanced clinical stage with high mortality and little response to chemotherapy. Patients with primary CNS histiocytic sarcoma described in case reports have expired within 3 to 8 months of diagnosis from various complications. ${ }^{3,4}$

Our patient presented with only extranodal involvement of the CNS, suffered from severe neurological impairment and survived for one month after her diagnosis was made. Although she likely died from complications of lung cancer, her functional status was progressively worsening due to the increasing size of the histiocytic sarcoma in her brain.

Due to the rarity of the disease and lack of studies to validate diagnostic criteria, HS is difficult to diagnose. Several markers used to help diagnose $\mathrm{HS}$ are histiocyte-associated antigens, such as CD68, lysozyme, CD11c, and CD14. ${ }^{2,4}$ Diagnosis also requires a lack of expression of other known cell lineages such as B-cells, T-cells, myeloid cells, follicular dendritic cells, and CD30. ${ }^{4}$ More recently, CD163 has been identified as an important factor for the diagnosis of HS. CD163 is a hemoglobin scavenger receptor and its expression is limited to neoplasms of monocytic/ histiocytic derivation. It is more specific than other markers such as CD68. Cao et al. published in 2007 a case of primary CNS HS and confirmed the importance of distinguishing CD68 and CD163 positive tumors as HS versus other malignancies. ${ }^{6}$ Before CD163 was identified, HS was more difficult to diagnose and perhaps overdiagnosed. ${ }^{1}$ There are several case reports identifying histiocytic sarcoma as a primary CNS tumor ${ }^{4,5}$, but these reports came before identification of CD163 as an important factor for diagnosis. The patient presented in the case above had a primary CNS malignancy that stained positive for both histiocyte-associated antigen CD68 and histiocyte-specific CD163 receptor.
Cao et al. also commented that, considering their findings of mild cytologic atypia and a profound inflammatory componont, their initial impression was one of a chronic inflammatory process. ${ }^{6}$ Prominent inflammatory infiltrates have been described in all five of the previously reportedpublished cases in the literature of primary CNS HS. A chronic inflammatory process and was on our differential diagnosis in the beginning as well. ${ }^{6}$ Interestingly, due to the rarity of the final diagnosis, which required numerous histochemical stains and second opinions from pathology departments at other institutions, the final diagnosis of histiocytic sarcoma was not obtained for several weeks following the biopsy. During this time, other possible etiologies for the brain lesion, including infectious diseases, were ruled outconsidered and eventually eliminated.

There radiographic appearance of CNS lesions is the key to their differentiation. Features typical of metastatic disease include multiple lesions, spherical shape, well demarcated borders, and their location at the gray matter-white matter junction. ${ }^{7}$ The lesion in this case, although well-circumscribed, was felt to be uncharacteristic of metastatic disease due to the depth of location and lack of multiple CNS lesions. Ultimately, it was this radiographic appearance that lead us to pursue further pathologic diagnosis.

\section{References}

1. Vos JA, Abbondanzo SL, Barekman CL, et al. Histiocytic sarcoma: a study of five cases including the histiocyte marker CD163. Modern Pathology 2005;18, 693-704.

2. World Health Organization Classification of Tumors: Pathology and Genetics of Tumours of Haematopoietic and Lymphoid Tissue. Lyon France IARC Press; 2001:278-279.

3. Yu L, Yang SJ. A Case of Primary Histiocytic Sarcoma arising from the Thyroid Gland. Pathology and Oncologic Research 2009; 1219-4956.

4. Cheuk W, Walford N, Lou J, et al. Primary Histiocytic Lymphoma of the Central Nervous System. The American Journal of Surgical Pathology 2001;25: 1372-1379

5. Sun W, Nordberg M, Fowler M. Histiocytic Sarcoma Involving the Central Nervous System. Ther American Journal of Surgical Pathology 2003;27: 258-265.

6. Cao M, Eshoa C, Schultz C, et al. Primary Central Nervous System Histiocytic Sarcoma with Relapse to Mediastinum. Archives of Pathology Lab Medicine 2007;131: 301-305.

7. Sagar Stephen M, Israel Mark A, "Chapter 374. Primary and Metastatic Tumors of the Nervous System” (Chapter). Fauci AS, Braunwald E, Kasper DL, Hauser SL, Longo DL, Jameson JL, Loscalzo J: Harrison's Principles of Internal Medicine, 17e: http://www.accessmedicine.com/content.aspx?aID=2906281. Accessed October 27, 2009 